Indonesian Journal of Physics

Vol 19 No. 3, July 2008

\title{
A Preliminary Result of Seismoelectric Responses Study on Shallow Fluid-Saturated Layer: Numerical Modeling Using Transfer Function Approach
}

\author{
Alamta Singarimbun, Harry Mahardika, Wahyu Srigutomo, and Umar Fauzi \\ Physics of Complex System Research Division \\ Faculty of Mathematics and Natural Scienses \\ Institut Teknologi Bandung \\ e-mail:alamta@fi.itb.ac.id
}

\begin{abstract}
Seismoelectric phenomena occurs when a seismic wave propagating in a fluid-saturated medium and crossing an interface of different fluid-saturated mediums that induces electrical field or cause radiation of an electromagnetic wave where only the electric part is being measured. In this paper, we present our research progress on seismoelectric imaging of shallow fluid-saturated layers. The progress mainly discusses on our finite-difference algorithm which provides an alternative method to simulate seismic wave propagation in fluid saturated porous media generating electric fields through an electrokinetic mechanism called seismoelectric coupling. The target of this numerical simulation is to see whether the seismoelectric responses of coseismic field and interface boundary effect can be image on the seismoelectrogram. The simulation begins with the calculation of seismic displacement by using the classical homogeneous formulation. Next, we calculate the seismoelectric responses using the seismoelectric transfer function. The algorithm is then used to simulate seismoelectric responses created from various conditions of finite-infinite and finite-infinite sand-clay model, surface to downhole and crosshole configurations.
\end{abstract}

Keywords : Seismoelectric phenomena, Fluid-saturated layers, Electrokinetic mechanism, Seismoelectric coupling, Interface boundary effect, Finite-infinite, Finite-infinite

\subsection{Introduction}

Geophysical methods provide an affordable means to determine useful information about the subsurface without digging holes or drilling wells. However, the existing geophysical techniques only provide very little information about hydraulic permeability. A thin $(\sim 1 \mathrm{~cm})$ impermeable layer has a major impact on ground water flow, and also an interest in environmental studies, but it is virtually invisible to conventional geophysical methods. Another example of a thin highly-permeable fracture is also a great interest, but is equally difficult to image. To fill these weaknesses, we study a natural phenomenon called seismoelectric phenomenon that created from a sophisticated mechanism called electrokinetic effect. We focused only to this effect because of its potential applications in geophysical prospecting. The seismoelectric method, or measurements of seismoelectric responses created from the electrokinetic effect in fluid-saturated medium, can potentially provide information about subsurface permeability contrasts including very thin layers.

Generally, our research is divided to two stages: numerical modeling and field measurements. While subsurface imaging is mainly depends on field measurement, many of the potential benefits and limitations of seismoelectric prospecting can be addressed through numerical simulation. Many examples of numerical simulation are provided by authors like Haartsen and Pride $^{1)}$ who present a seismoelectric modeling algorithm for full threedimensional (3D) wave propagation through onedimensional (1D) stratified geologic models of both lithological and fluid-chemistry contrasts/interfaces. Next, Garambois and Dietrich ${ }^{2)}$ use a similar algorithm to test seismoelectric sensitivity to contrasts in porosity, permeability, salinity, and pore-fluid viscosity. Because most realistic applications of the seismoelectric method involve complicated subsurface geometries, grid-based modeling algorithms to simulate realistic applications of the method are needed. Such modeling capabilities will help provide synthetic data sets for designing and testing dataprocessing algorithms. This was done by Haines and Pride $^{3)}$, who present a two-dimensional (2D) finite difference (FD) modeling algorithm that simulates seismoelectric phenomena in earth models with arbitrary heterogeneity and geometry variations (layer thickness, lateral extent, and lateral position) on the observed seismoelectric response, but allows for all poroelastic wave modes (fast waves, slow waves, and shear waves). However, the calculations required for each seismoelectric simulation are very costly. So, an alternative approaches on numerical simulation are needed to be introduced and applied. To compromise this problem, we develop a seismoelectric modeling algorithm that directly calculate the electric field from seismic displacement using the seismoelectric transfer function $^{4)}$. 


\section{Electrokinetic Effect}

Electrokinetic effect is associated with the rise of electric or electromagnetic responses due to relative change of fluid phase into solid phase ${ }^{5)}$. In the earth, electrokinetic effects occur in fluid-saturated porous rocks. Microscopically, these effects are due to adsorption of negatively charged ions in matrix rocks that in turn causes the excess of free positive ions in rock fluid. In other words, molecular charge separation occurs at matrix-fluid boundaries which are also called the electric double layer ${ }^{6}$. The separation is caused by propagation of seismic waves in rocks that gives rise to relative displacement of ion carrying fluid with respect to the rock matrix, enabling streaming electric current to flow. This current will produce further separation in macroscopic scale which becomes the source of induced electric field or electromagnetic wave radiation. Properties of the field are influenced by electrochemical properties of the matrix-fluid boundary and mobility of ions in fluid, types of fluid (e.g. water, air, or oil), types of matrix (clastic or carbonate), and mechanical and structural properties of the rocks (elastic moduli, porosity, permeability, and saturation).

The governing equations of a coupled electromagnetic-acoustic wave propagating in anisotropic and heterogeneous medium ${ }^{6}$, are written as

$$
\begin{aligned}
& -\omega^{2} \rho \vec{u}=-\vec{\nabla} P_{C}+\vec{\nabla} \cdot \vec{\tau}^{D}+\omega^{2} \rho_{f} \vec{w}, \\
& \vec{\tau}^{D}=G\left(\vec{\nabla} \vec{u}+\nabla \vec{u}^{T}-\frac{2}{3} \vec{\nabla} \cdot \vec{u} \vec{I}\right), \\
& -\left[\begin{array}{c}
P_{C} \\
p_{f}
\end{array}\right]=K_{U}\left[\begin{array}{cc}
1 & B \\
B & B / \alpha
\end{array}\right]\left[\begin{array}{c}
\vec{\nabla} \cdot \vec{u} \\
\vec{\nabla} \cdot \vec{w}
\end{array}\right], \\
& {\left[\begin{array}{c}
-i \omega \vec{w} \\
\vec{J}
\end{array}\right]=\left[\begin{array}{cc}
\mathrm{k}(\omega) / \eta_{f} & \mathrm{~L}(\omega) \\
\mathrm{L}(\omega) & \sigma(\omega)
\end{array}\right]+\left[\begin{array}{c}
-\nabla p_{f}+\omega^{2} \rho_{f} \vec{u} \\
\vec{E}
\end{array}\right]} \\
& \vec{\nabla} \times \vec{H}=\vec{J}-i \omega \varepsilon \vec{E},
\end{aligned}
$$

and

$$
\vec{\nabla} \times \vec{E}=i \omega \mu \vec{H}
$$

The deviatoric stress tensor $\vec{\tau}^{D}$ is defined as $\vec{\tau}^{D}=\vec{\tau}+P_{C} \vec{I}$ where $\vec{\tau}$ is the total bulk stress tensor acting on the material, and $P_{C}$ is the confining (or total bulk) pressure, while $\vec{I}$ is the identity matrix. Others are: seismic displacement in solid phase $\vec{u}$, relative fluid-solid motion $\vec{w}$, medium's bulk density $\rho_{\mathrm{B}}$, pore-fluid viscosity $\eta$, Gassmann moduli $K_{U}$, Skempton's coefficient $B$, Biot and Willis constant $\alpha$, shear moduli $G$, and $\vec{J}, \vec{D}, \vec{E}, \vec{H}, \vec{B}, \vec{C}$ are the electric current density, electric displacement, electric field, magnetic field, magnetic induction, and electrical source term respectively. On the other hand, electric conductivity $\sigma(\omega)$, permeability $k(\omega)$ and electrokinetic coupling coefficient $L(\omega)$, are defined as frequency dependent functions. The electrokinetic coupling coefficient $L$ is a key component of these equations, as it describes the relation between grain/fluid motion and electric fields. If $L$ is set to zero, these equations decouple into Biot's and Maxwell's equations. By using a plane-wave solution of the governing equations, Pride and Haartsen ${ }^{6}$ derive the elementary displacement and EM fields (eigen response) of an isotropic and homogeneous wholespace. Using their results, Garambois and Dietrich $^{4)}$ express the electric field $\vec{E}$ as a function of the seismic displacement $\vec{u}$ and demonstrate that lowfrequency approximations of these relationships may be written in the form

$$
\vec{E}=\frac{1}{\sigma_{f}} \frac{\varepsilon_{o} \rho_{f}{ }^{\kappa} f}{\eta}\left(1-\frac{\rho}{\rho_{f}} \frac{C}{H}\right) \frac{\partial^{2} \vec{u}}{\partial t^{2}}
$$

Equation 7 is called as the seismic to electric field (seismoelectric) transfer fuction in low-frequency regime; where it is valid in the diffusive regime, i.e., when conduction currents dominate displacement currents.

For fluid-saturated homogeneous-isotropic rocks, spherical seismic waves from a point source will propagate in all directions instigating cation-anion separations along the half wavelength of the waves. The separations continuously occur as long as the seismic waves propagate in the medium, and will be detected at the surface in time when the waves reach the dipole antennas. Electric signal triggered by this conversion mechanism is called coseismic field since the electric field propagates in the same time as the seismic wave, as displayed in Figure 1. The travel time of seismoelectric waves is almost similar to the seismic ones. The second mechanism is known as interface response or boundary interface response. It is created when seismic waves arrive at an interface of two media with vertically fluid saturation contrast. Because of pressure difference in the two parts of the interface in the first Fresnel zone, imbalance of streaming current in the upper part of the interface will initiate the separation of charges in the lower part. The formed electric dipoles will radiate electromagnetic waves which is measurable at the surface. In this mechanism, the seismoelectric waves propagate from the interface to the surface. They take shorter travel time than the seismic waves which have to experience two-way travel time from source to receiver.

The second mechanism occurs when P-wave encounters an interface in material properties (elastic, chemical, flow-properties). The charge separation in the wave is disturbed, causing asymmetry in the charge distribution, and resulting in what can be approximated as an oscillating electric dipole with its dominant contribution coming from the first seismic Fresnel zone ${ }^{1,7)}$. In other words, the entire region of the first Fresnel zone acts as a disk of vertical electric dipoles. Since the electromagnetic wavelengths at 
seismic frequencies are typically enormous compared to the depth of the interface, induction effects can be neglected, and the electric fields generated at the interface can be modeled as being quasi-static. The resulting electric field distribution is that of a quasistatic dipole. This field, shown in Figure 2, is called as "interface response"; where it can be measured almost immediately at the Earth's surface since the travel-time of electromagnetic radiation is negligible compared with that of seismic waves $\left(\mathrm{v}_{\mathrm{EM}} \sim 10^{5} \mathrm{v}_{\mathrm{P}}\right)$. Unlike the coseismic field, which contains information only about the material immediately surrounding the receiver dipole, the interface response can provide useful new subsurface information. In particular, the interface response occurs even for very thin layers $(\sim$ $10^{-2} \mathrm{~m}$ ), such as thin fractures in solid rock, or a thin impermeable layer in an aquifer or reservoir.

In the case of two horizontal layers a seismoelectric data (seismoelectrogram), collected with geometry similar to conventional seismic recording, include simultaneous recordings of the interface response from subsurface layers and coseismic fields. The direct field may also be recorded along with the other two forms of seismoelectric response, but these occur only for a brief period after time zero so do not present a problem in surveys targeting layers deeper than a few meters. The coseismic fields and interface response are of comparable magnitude when the recording antennas are within a seismic wavelength of the interface. However, since the interface response field is essentially a dipole, its magnitude is decreasing as the distance for receivers from the interface increased and can thus be many orders of magnitude smaller than the coseismic fields, especially as target depth increases. Coseismic energy therefore represents a formidable form of coherent source-generated noise that can obscure large parts of the seismoelectric record. An example of seismoelectrogram consisting the coseismic fields, interface boundary, and other efeect can be seen in Figure 3.

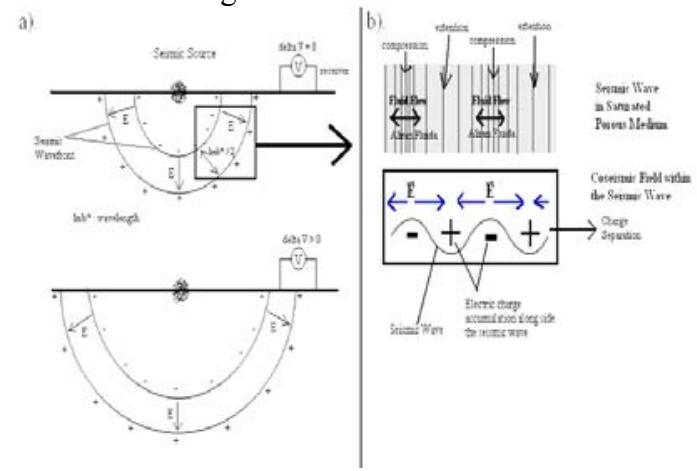

Figure 1. Electrokinetic conversion caused by seismic wave propagation in homogeneous porous medium, generating coseismic field. a). Coseismic field recorded on the surface after seismic wave propogates underneath the receiver. b). Connection between seismic wave and coseismic fields.

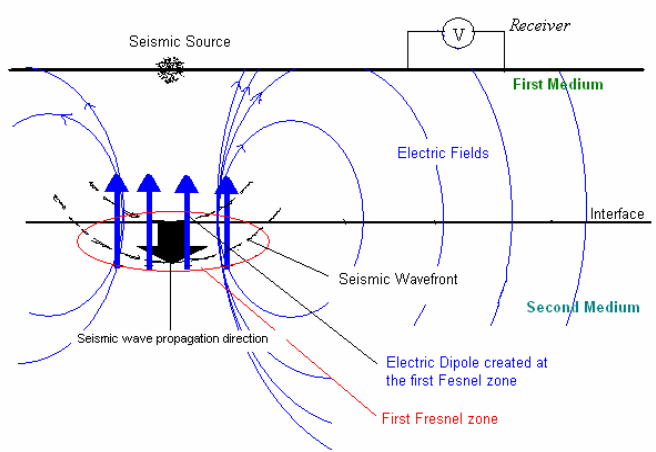

Figure 2. Interface boundary response or electrokinetic conversion at an interface happens when seismic wave crosses the layers boundary and act as the first Fresnel zone generating electric field response. Due to its dipole behavior, the electric field propagation, generated from this mechanism, is faster than a twoway travel seismic wave.

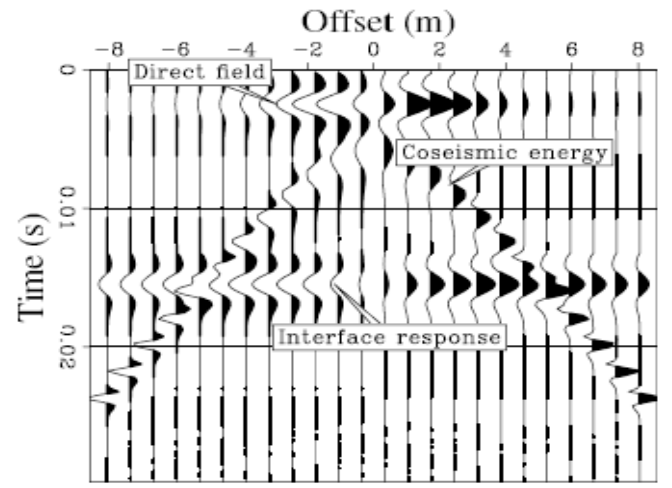

Figure 3. A typical image of synthetic or recorded seismoelectrogram containing three patterns of electric fields: the coseismic field, interface response, and direct field ${ }^{7}$.

\section{Applying the Numerical Algorithm}

To begin the modeling, one should recall the seismic to electric field transfer function of equation (7). The equation explicitly shows that the seismic wavefield or displacement is decoupled from the electric field, so it can be simulated independently. Next, one can stated that a two dimensional seismic Pwave equation $^{8)}$ is described as :

$$
\begin{aligned}
& \left(\left(\frac{\partial^{2}}{\partial x^{2}}+\frac{\partial^{2}}{\partial z^{2}}\right)-\frac{1}{v_{p}^{2}} \frac{\partial^{2}}{\partial t^{2}}\right) u=0 \\
& v_{p}=\sqrt{\frac{\lambda_{U}+2 G}{\rho}}
\end{aligned}
$$

where $v_{p}$ is the velocity of the P-wave. The seismic Swave and surface wave are not included in this model, since both have their own transfer function ${ }^{4,6)}$. The seismic wave equation then can be approximated using finite difference method for hyperbolic/ wave equation. Hence, equation (8) can be discretisized into 


$$
\begin{aligned}
& \frac{\mathrm{u}_{\mathrm{i}+1, \mathrm{j}}^{t}-2 u_{i, j}^{t}+u_{i-1, j}^{t}}{\Delta x^{2}} \\
& +\frac{\mathrm{u}_{\mathrm{i}, \mathrm{j}+1}^{t}-2 u_{i, j}^{t}+u_{i, j-1}^{t}}{\Delta z^{2}} \\
& -\frac{1}{v_{p_{i, j}}^{2}} \frac{\mathrm{u}_{\mathrm{i}, \mathrm{j}}^{t+1}-2 u_{i, j}^{t}+u_{i, j}^{t-1}}{\Delta t^{2}}=0
\end{aligned}
$$

Solving for $\mathrm{u}_{\mathrm{i}, \mathrm{j}}^{t+1}$, one can get an explicit forward differenced $2 \mathrm{D}$ wave equation in time

$$
\begin{aligned}
\mathrm{u}_{\mathrm{i}, \mathrm{j}}^{t+1} & =2 u_{i, j}^{t}-u_{i, j}^{t-1}+v_{p_{i, j}}^{2} \Delta t^{2} \\
\times & \left(\begin{array}{l}
\frac{\mathrm{u}_{\mathrm{i}+1, j}^{t}-2 u_{i, j}^{t}+u_{i-1, j}^{t}}{\Delta x^{2}} \\
+\frac{\mathrm{u}_{\mathrm{i}, \mathrm{j}+1}^{t}-2 u_{i, j}^{t}+u_{i, j-1}^{t}}{\Delta z^{2}}
\end{array}\right)
\end{aligned}
$$

After the seismic wave has been modeled, the last step is calculating the electric field using the transfer function. Therfore equation (7) is discretisized into

$$
\begin{gathered}
E_{i, j}^{t}=\left(\frac{1}{\sigma_{f_{i, j}}} \frac{\varepsilon_{0} \kappa_{f_{i, j}} \rho_{f_{i, j}} \zeta_{i, j}}{\eta_{i, j}}\left(1-\frac{\rho_{i, j}}{\rho_{f_{i, j}}} \frac{C_{i, j}}{H_{i, j}}\right)\right) \\
\times\left(\frac{\mathrm{u}_{\mathrm{i}, \mathrm{j}}^{t+1}-2 u_{i, j}^{t}+u_{i, j}^{t-1}}{\Delta t^{2}}\right)
\end{gathered}
$$

Adding a point source into the algorithm, one can use an explosion effect ${ }^{7)}$ at the source point $\vec{r}_{s}$ using isotropic source $S$ term is added to equation (10) in the form of

$$
s(\vec{r}, t)=s(t) M_{o} \delta\left(\vec{r}-\vec{r}_{s}\right)
$$

where $s(t)$ is a dimensionless source wavelet with a peak amplitude of one and $M_{0}$ is the seismic moment release (units of energy). To model a Dirac delta function on a 2D finite-difference grid, with discretization lengths $\Delta_{i}$ in each of the $i$ directions, one will have

$$
\delta\left(\vec{r}-\vec{r}_{s}\right)=\delta\left(x-x_{s}\right) \delta\left(z-z_{s}\right) \approx 1 /\left(\Delta_{x} \Delta_{z}\right)
$$

at the point where the source is detonated and zero elsewhere.

Next, all of the physical properties needed for the simulation are defined. To simulate $1 / 2 \mathrm{~kg}$ shot of dynamite we used $M o=200 \mathrm{~J}^{3)}$. Electrokinetic coupling coefficient $\mathrm{L}$ is calculated using zeta potential $\varsigma=0.01+0.25 \log ^{10} C_{m}$; where $C_{m}$ is the salt concentration in moles/liter of the fluid saturating the pores. The pore fluid in all the examples is assumed to be water. For a sodium chloride electrolyte at room temperature, the electric conductivity of the fluid is $\sigma_{f}=10 C_{m}$, where $C_{m}$ again is the salt concentration in moles/liter. Medium's conductivity is given by $\sigma=\sigma_{f} / F$, where the formation factor $F=\phi / \alpha_{\infty}$, defined using Garambois and Dietrich approximation $^{4)}$. Other fluid properties such as $\varepsilon_{f}=\kappa_{f} \varepsilon_{o}, \eta_{f}, \rho_{f}$, and $K_{f}$ are given in table 1, where $\varepsilon_{0}=8.8541878 \times 10^{-12}$. The rock density is given by $\rho=\phi \rho_{f}+(1-\phi) \rho_{s}$, and the incompressibility of the individual solid grains $K_{s}$ for sand and clay are also listed in the table 1 . To determine the poroelastic moduli, Gassmann equations are used ${ }^{4)}$. So, the only material properties that are allowed to vary in the following examples are $\lambda_{U}, K_{S}, G, \phi, k_{0}$, and $C_{M}$.

Before the algorithm is applied for various physical conditions, we do some test to the algorithm in order to check its adaptability and validity or similarity with results from other author ${ }^{9)}$. So, we choose result of Hainess and Pride ${ }^{3)}$ as a comparator for the second test. The seismic source is modeled using a sinc function wavelet and the discretization parameters are $\Delta_{z}=0.05 \mathrm{~m}, \Delta_{x}=0.07 \mathrm{~m}$, and $\Delta_{t}=$ $0.0013 \mathrm{~s}$. These parameters are determined to follow the stability conditions for finite difference modeling described by Kelly et.al., ${ }^{10)}$. Modeling dimension used in the algorithm is $102 \times 102$ pixels and total time step is 350 . To reduce the effect of "numerical reflection" over the grid edges, an additional 20 pixels at the right and left part of modeled region are being used. Boundary conditions used in the simulation are only applied to the seismic modeling part, where free surface boundary condition is applied at $\mathrm{z}=1$, while left and right side of the boundary are using Neumann boundary condition. For electrical field/seismoelectric response calculation part no boundary conditions are applied. Although the test shows that the algorithm fail to image interface-boundary responses ${ }^{11)}$, one can try the algorithm to be applied in several geometrical cases, in order to image or characterized the coseismic field signatures and the interface-boundary responses.

Table 1. Physical properties of sand and clay

\begin{tabular}{|l|l|l|}
\hline Properties & Sand & Clay \\
\hline$\lambda_{U}(\mathrm{GPa})$ & 6.626 & 12.403 \\
\hline$K_{s}(\mathrm{GPa})$ & 35 & 25 \\
\hline$K_{f}(\mathrm{GPa})$ & 2.27 & 2.27 \\
\hline$K_{f r}(\mathrm{GPa})$ & 0.3 & 0.3 \\
\hline $\mathrm{G}(\mathrm{GPa})$ & 0.35 & 0.23 \\
\hline$\eta(\mathrm{Pa} \mathrm{s})$ & $1 \times 10^{-3}$ (water) & $1 \times 10^{-3}$ (water) \\
\hline$\phi(\%)$ & 30 & 10 \\
\hline$k_{0}\left(\mathrm{~m}^{2}\right)$ & $10 \times 10^{-11}$ & $10 \times 10^{-16}$ \\
\hline$\rho_{b}\left(\mathrm{~kg} / \mathrm{m}^{3}\right)$ & 2600 & 2600 \\
\hline$\rho_{f}\left(\mathrm{~kg} / \mathrm{m}^{3}\right)$ & $1000($ water $)$ & $1000($ water $)$ \\
\hline$\kappa_{f}$ & $80($ water $)$ & $80($ water $)$ \\
\hline$C_{m}(\mathrm{moles} / \mathrm{L})$ & $1 \times 10^{-4}$ & $5 \times 10^{-4}$ \\
\hline
\end{tabular}

Four cases are determined: 1 . horizontal finite to infinite block layer case, 2. dipping set of block 
layers which resembling a dipping layer case, 3 . downhole / VSP case, and 4. crosshole case. For the first case, a finite length and thickness of a block target layer are expanded gradually in such a ways to eventually becoming a half-spaced target layer. This is on the $84 \mathrm{~cm}$ times $20 \mathrm{~cm}$ block case, where amplitude variation the reflected and diffracted coseismic field will be compared. Other target layer geometry and its environment are showed in the configuration of figure 4. The second case is a development from the blocklayer model. It resembles a dipping horizontal layer, that often in FD modeling is not be able to smoothly model. In this case, as one can see the configuration in figure 5 , one block is characterized by its $0.5 \mathrm{~m}$ thickness times the $1.4 \mathrm{~m}$ length. Then, six other blocks are placed under the first but gradually spaced $0.7 \mathrm{~m}$ to the right direction. So, the target consist of seven dipping block layer, representing a dipping layer with an angle of $\theta=32^{\circ}$. The third geometry is similar as the finite-thickness horizontal layer model, but the difference is that the receivers are placed not in the surface but on a vertical borehole, as can seen in figure 6 . The borehole offset is at $0.5 \mathrm{~m}$ from the source point, while clay aquitard has $2 \mathrm{~m}$ thickness at $2 \mathrm{~m}$ depth. The application of seismoelectric modeling inside a borehole is potentially useful in addition to downhole or vertical seismic profiling (VSP) measurement, especially if one would like to see a very thin layer, reservoir monitoring, or fractures within the subsurface. The last model is another development from the downhole-VSP model, where the source point is placed under another borehole which has a lateral distance of $4 \mathrm{~m}$, as seen in figure 7 .

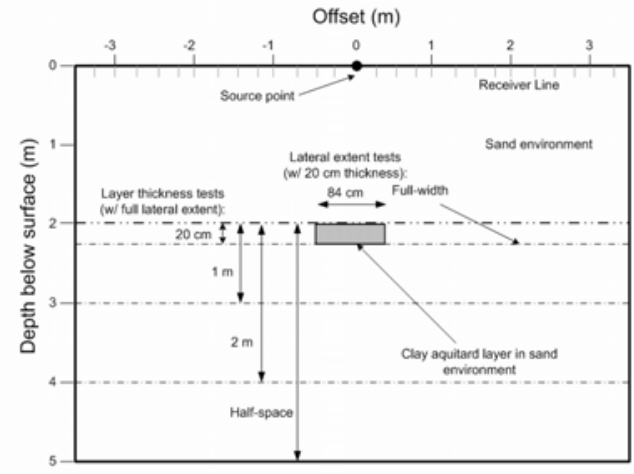

Figure 4. Model of a finite to infinite clay aquitard subsurface layer in sandy environment. The target is situated at $2 \mathrm{~m}$ depth, while the thickness and lateral extent are varies.

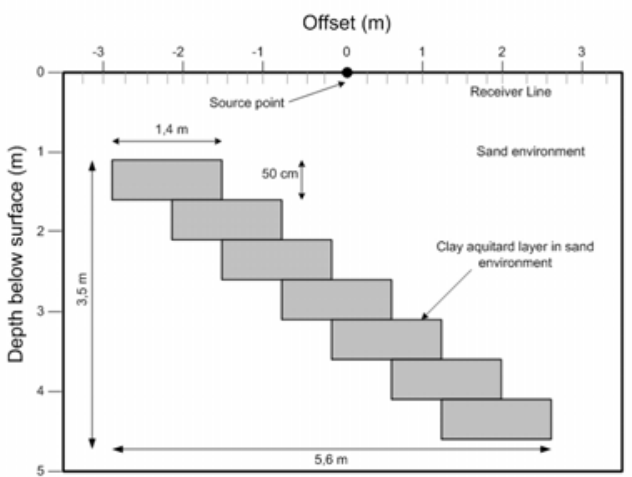

Figure 5. Model of dipping blocks of clay layer, resembling a $32^{0}$ dipping horizontal layer, over the sand environment.

The depth of source point borehole can be similar to receiver's borehole, but in this model the depth are only $3 \mathrm{~m}$. Clay target used in this model is a four squared meter block, situated between the source point and the receivers and $2 \mathrm{~m}$ below the surface. Similar to the application of downhole-seismoelectric modeling, this simulation is potentially useful to see a very thin layer, reservoir monitoring, or fractures within the subsurface.

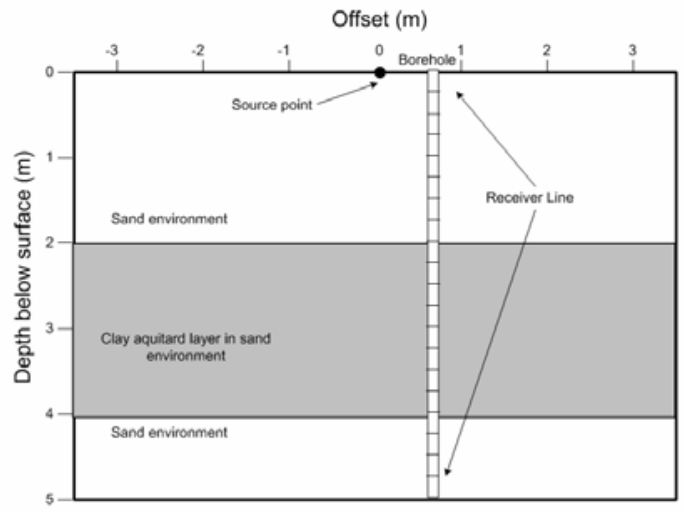

Figure 6. Model used in downhole-seismoelectric response modeling. The target layer is $2 \mathrm{~m}$ thick horizontal layer situated at $2 \mathrm{~m}$ depth. Receivers are place on the borehole while its offset is $0.5 \mathrm{~m}$ beside the source point. 


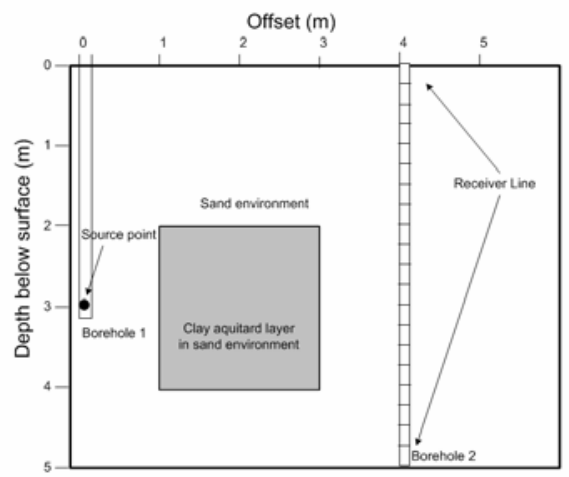

Figure 7. Model used in crosshole-seismoelectric response modeling. The target layer is $4 \times 4 \mathrm{~m}^{2}$ clay block situated at $2 \mathrm{~m}$ depth. Receivers are place on another borehole with offset distance of $4 \mathrm{~m}$.

The results are then explained. First a synthetic seismoelectrogram shown in figure 8 is a simulation result for the $20 \mathrm{~cm} \mathrm{x} 84 \mathrm{~cm}$ length clay aquitard case. This image shows the strongly direct coseismic arrivals, as well as the flat reflected coseismic event from the clay aquitard. From this image one can also see the dimensions of the target where the top and bottom of the target are visible at $180 \mathrm{~ms}$ and $280 \mathrm{~ms}$ respectively. Next, the case of $20 \mathrm{~cm}$ and $1 \mathrm{~m}$ thick full length clay aquitard is explained. Both are presented in figure 9 and figure 10 respectively, and the direct and two reflected coseismic arrivals are clearly visible. The differences are that the top and bottom reflection of the $20 \mathrm{~cm}$ thick model is at 180 $\mathrm{ms}$ and $190 \mathrm{~ms}$ respectively, while the $1 \mathrm{~m}$ thick model is at $180 \mathrm{~ms}$ (top boundary) and $250 \mathrm{~ms}$ (bottom boundary). This mean that layer's thickness determination using reflected coseismic field is able to be done with this algorithm, although determination method using interface response is more preferable. For the next case, coseismic direct field is clearly visible at the upper part of the seismoelectrogram, as shown in figure 11. The reflection of the coseismic field, however, is not so smoothly as before. The only reflection that visible is the event from the top block, and events of top and bottom reflections from other 6 blocks are difficult to recognize. Although blurred, the diffraction trends are positive, following the trends of dipping modeled layer. And the angle of the dipping trends is relatively same with the model, i.e., $\theta \sim 30^{\circ}$. This application shows that dipping layer imaging using reflected coseismic field is able to be done with this algorithm. And since there are no references on seismoelectric modeling of dipping layer, this should be the foundations for further research. The last two cases, is a development of this algorithm. Reference works on seismoelectric modeling of downhole and crosswell configuration mainly focus on the measurements experiment ${ }^{11)}$, while the numerical modeling is only few ${ }^{1-3)}$. So, the creation of transfer function numerical algorithm is trying to add more references on this topic, in hope that this will be useful for further development. Even though coseismic field responses are can not be certainly interpreted from both results, as one can see in figure 12 and figure 13, the indicators for good modeling results are visible. First, the seismoelectric response from downhole configuration shows direct coseismic responses that are clearly at sand, but weaken at clay. Then, the seismoelectric response from crosswell configuration shows diffractions pattern that image the thickness of the target layer. In other word, there is a lot works need to be done if one would like to develop this topic.

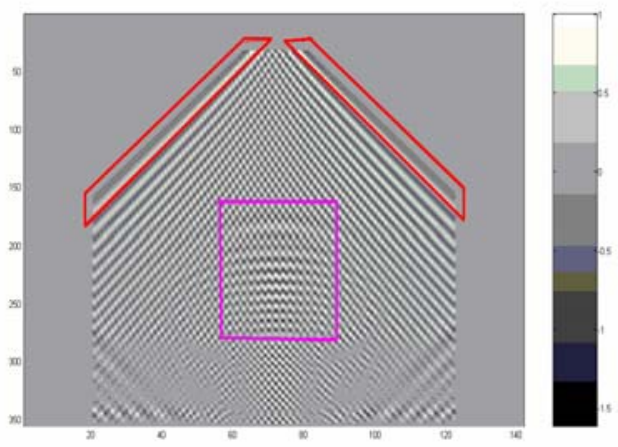

Figure 8. Synthetic seismoelectrogram of an $84 \mathrm{~cm}$ length and $20 \mathrm{~cm}$ thick clay block at $2 \mathrm{~m}$ depth of a sandy environment. The reflected and diffracted (pink box) coseismic fields are clearly visible at $180 \mathrm{~ms}$.

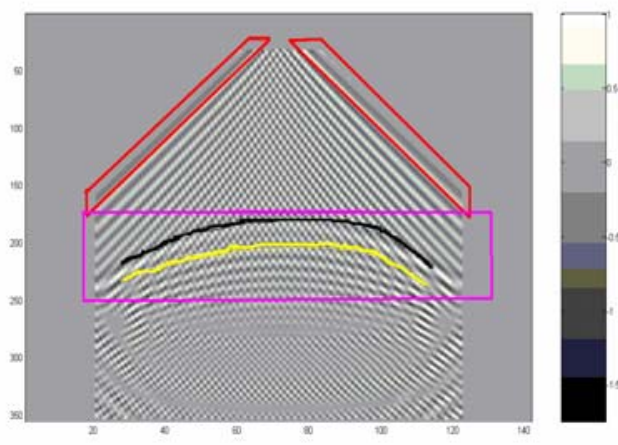

Figure 9. Synthetic seismoelectrogram of a $20 \mathrm{~cm}$ thick clay layer at $2 \mathrm{~m}$ depth of a sandy environment. The reflected (pink box) coseismic responses are clearly visible, where the reflections characterize the top boundary layer at $200 \mathrm{~ms}$ (black) and bottom boundary at $210 \mathrm{~ms}$ (yellow). 


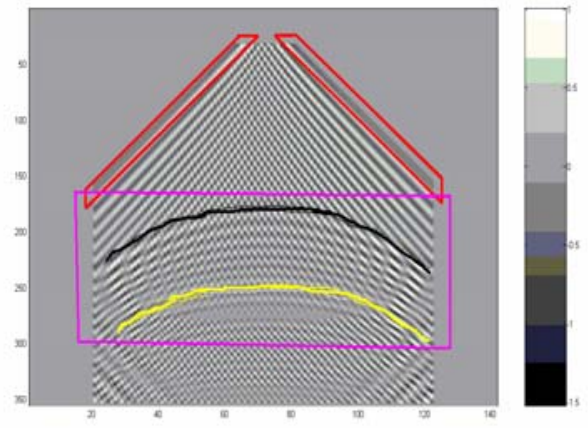

Figure 10. Seismoelectrogram of a $1 \mathrm{~m}$ thick clay layer at $2 \mathrm{~m}$ depth of a sandy environment. The reflected (pink box) coseismic responses are clearly visible, where the reflections characterize the top boundary target at $200 \mathrm{~ms}$ (black) and bottom boundary target $250 \mathrm{~ms}$ (yellow).

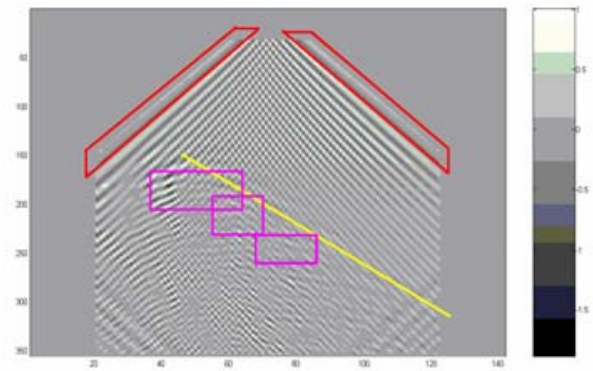

Figure 11. Synthetic seismoelectrogram of a dipping clay layer in sand environment. The direct (red box) coseismic response is clearly visible. And also the reflected field (pink boxes), where the signatures are not smoothly as before but gradually dipping to the right side (yellow). Top and bottom boundary characterization for each layer are difficult to characterize since diffractions from bottom layers are many.

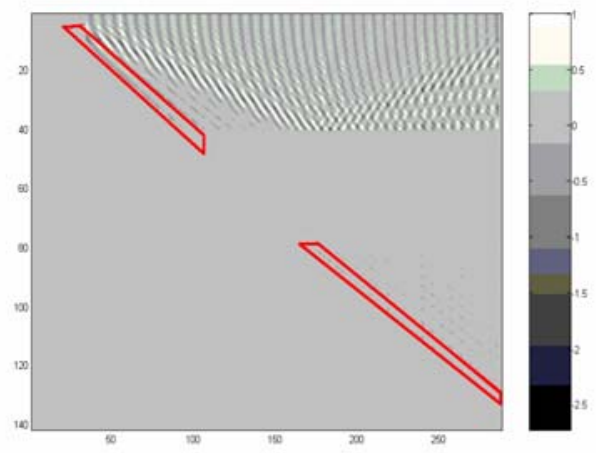

Figure 12. Seismoelectrogram of a $2 \mathrm{~m}$ thick clay layer at $2 \mathrm{~m}$ depth of a sandy environment. The direct (red box) coseismic responses are clearly visible at sand, but weaken at clay. Reflections of upgoing and downgoing wave that typical of this configuration are hard to identify.

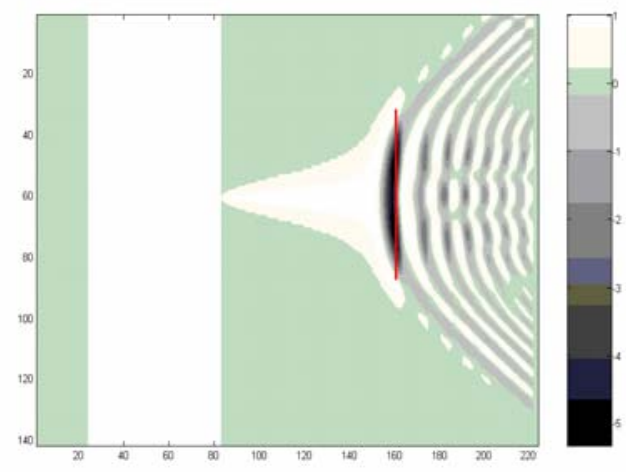

Figure 13. Seismoelectrogram of a $4 \mathrm{~m}^{2}$ block of clay at $2 \mathrm{~m}$ depth of a sandy environment. The reflected coseismic responses and its diffractions are clearly visible, and the thickness of the target is exactly obtained (red). 4 .

\section{Discussion}

The numerical modeling presented in this paper is an alternative method on seismoelectric simulation to be performed on a grid. The algorithm allows one to simulate targets and heterogeneity having arbitrary $2 \mathrm{D}$ heterogeneity. It thus simulates applications of the seismoelectric method with realistic subsurface heterogeneity. Such simulations will be valuable as we attempt to determine the best applications for the seismoelectric method. The modeling results stated that the transfer function numerical algorithm can image layers from the arrival of reflected coseismic field. However, the failure of this algorithm on simulating the interface response is a disadvantage. And the adaptability of the algorithm to detect physical properties changes is also questioned. However, the dipping layer simulation gives potential for the seismoelectric method to provide useful information that is otherwise unavailable.

\section{Conclusions}

These results encourage us to continue more advanced numerical simulation and provide guidance in experimental design. In particular, we would highlight the value of creative survey geometries for the downhole, crosswell, and more-advance dipping layer model geometry used for examples. Fullequation numerical modeling in FD scheme is worth to done, in order to being applied on deeper geophysical targets. Lastly, further treatments for all of the transfer function algorithm and its results, using data processing sequences, are also supported. So, the study of seismoelectric phenomena, especially on its numerical simulation, will be more flourish with alternatives approach.

\section{Acknowledgements}

We would like to send our gratitude to the Ministry of Research and Technology Republic of Indonesia for funding us in our research on 
seismoelectric imaging and numerical modeling of shallow hidro-thermal layer, under the guidance of Program Insentif Riset grant. And we would also like to thank ITB for supporting our prvious works on application of seismoelectric imaging and modeling of shallow subsurface characterization, under the guidance of PHK-B grant. Besides all difficulties and the relatively slow progress that we face at the past, we hope that with this grant our research will be successful and methods that we develop will be able to be applied extensively.

\section{References}

1. M.W. Haartsen, and S.R. Pride, Electroseismic Waves from Point Sources in Layered Media, J. Geophys. Res. 102, 24745-24769, 1997.

2. S. Garambois, and M. Dietrich, Full Waveform Numerical Simulations of Seismoelectromagnetic Wave Conversions in Fluid-Saturated Stratifed Porous Media, J. Geophys. Res. 107, JB000316, 2002.

3. S. Haines, and S.R. Pride, Seismoelectric Numerical Modeling on A Grid, Geophysics 71, N67-N65, 2006.

4. S. Garambois, and M. Dietrich, Seismoelectric Wave Conversions in Porous Media: Field Measurements and Transfer Function Analysis, Geophysics 66, 1417-1430, 2001.
5. S. Glasstone, An Introduction to Electrochemistry, Reprint 2002, Affiliated East and West Press, New Delhi, 521-544, 1942.

6. S.R. Pride, and M.W. Haartsen, Electroseismic Wave Properties, Journal of Acoustical Society of America 100, 1301 - 1315, 1996.

7. S. Haines, Seismoelectric Imaging of Shallow Targets, Ph.D. dissertation, Stanford University, 2004. http://sep.stanford.edu.

8. R.E. Sheriff, and L.P. Geldart, Exploration Seismology, Volume I, Cambridge University Press, Cambridge, 1982.

9. H. Mahardika, and A. Singarimbun, Numerical Modeling of Seismoelectric Responses: Transfer Function Approach, Proc. Asian Physics Symposium (APS) Bandung, 2007.

10. Kelly, et. al., Synthetic seismograms: a Finitedifference Approach, Geophysics 41, 2-27, 1967.

11. H. Mahardika, and A. Singarimbun, Electrokinetic Dffect in Seismoelectric Phenomena: a Research of Physics of Complex System Group Institute of Technology Bandung, Int. Workshop on Seismo-Electromagnetism Phenomena (IWSEP) 2007, Bandung, November 2007.

12. Z. Zhu, M.W. Haartsen, and M.N. Toksoz, Experimental Studies of Electrokinetic Conversions in Fluid-Saturated Borehole Models, Geophysics 64, 1349-1356, 1999. 